\title{
CORRELATION BETWEEN CA PLAGES AND LONGITUDINAL MAGNETIC FIELDS OF THE CSSAR ACTIVE REGIONS
}

\author{
V. BUMBA \\ and \\ G. GodoLI \\ (The Astronomical Institute of the \\ Czechoslovak Academy of Sciences, \\ Ondřejov, C.S.S.R.) \\ (Catania Astrophysical Observatory, \\ Italy)
}

\section{A BST RACT}

The shapes of $\mathrm{K}_{2,3,2} \mathrm{Ca}$ plages and longitudinal magnetic fields are compared for CSSAR active regions during their first transit on the solar disk.

The bipolar magnetic regions follow the Hale polarity law.

Often the region of inversion of the magnetic field corresponds to a gap in the Ca plage structure.

Bright patches of plages may coincide with magnetic inclusions, magnetic hills, and occasionally also with regions of inversion of the magnetic field. The outline of Ca plages follow well the isogauss of $20-40$ oersted.

Histograms of the distances of individual magnetic field intensity peaks do not only correspond to the geometry of the supergranular network but also seem to indicate a difference in the organization of these peaks between the leading and following polarities.

\section{Introduction}

The existence of a high correlation between $\mathrm{Ca}$ plages and longitudinal magnetic fields is well established (Howard, 1959; Tsap, 1967).

Notwithstanding that, we have found it interesting to perform a comparison for the CSSAR Active Regions (AR) between Ca plages and longitudinal magnetic fields using the observational material collected at the Meudon Solar Service.

(a) For the Ca plages we had at our disposal a standardized file of at least one negative $\mathrm{K}_{2,3,2}$ heliogram per day, with a solar image diameter of $63 \mathrm{~mm}$ (the size of the Arcetri spectroheliograms), available at the Meudon Observatory for the CSSAR period 1965, March 11 - December 1 (Godoli and Monsignori Fossi, 1968).

(b) For the magnetic fields we had at our disposal a standardized file of Crimea or Meudon magnetic maps with the scale $1 \mathrm{~mm}=1$ " also available at the Meudon Observatory.

The agreement between Crimea and Meudon magnetic maps is fairly good. The time of observation of the magnetic maps are reported in Table 1.

Unfortunately, all the Crimea and Meudon magnetic material refers only to the first transit of the AR.

We also had at our disposal Mount Wilson small-scale magnetic maps. 
Table 1

Magnetic material available from Crimea and Meudon

\begin{tabular}{|c|c|c|c|c|c|c|c|c|c|}
\hline AR & $\begin{array}{l}\mathrm{Da} \\
196\end{array}$ & & $\begin{array}{c}\text { Time of } \\
\text { observation }\end{array}$ & Observatory & AR & $\begin{array}{l}\mathrm{Da} \\
196\end{array}$ & & $\begin{array}{c}\text { Time of } \\
\text { observation }\end{array}$ & Observatory \\
\hline 1 & Marcl & & $0730-1025$ & $\mathrm{C}$ & 7 & July & 12 & 1025 & $\mathbf{M}$ \\
\hline & & 18 & $1205-1310$ & $\mathrm{C}$ & & & 12 & 1200 & $\mathbf{M}$ \\
\hline & & 18 & $1315-1421$ & $\mathrm{C}$ & & & & & \\
\hline & & 19 & $0640-0750$ & C & 8 & Sept. & .04 & $1155-1345$ & $\mathrm{C}$ \\
\hline & & 19 & $1000-1115$ & $\mathrm{C}$ & & & 05 & $0537-0710$ & $\mathrm{C}$ \\
\hline & & & & & & & 05 & $0945-1100$ & $\mathrm{C}$ \\
\hline 3 & May & 16 & $0810-0953$ & $\mathrm{C}$ & & & 06 & $0535-0755$ & $\mathrm{C}$ \\
\hline & & 19 & $1040-1122$ & $\mathrm{C}$ & & & 06 & $0820-1100$ & $\mathrm{C}$ \\
\hline & & 20 & 0805 & $\mathbf{M}$ & & & 06 & $0820-1100$ & \\
\hline & & 21 & $0855-1000$ & $\mathrm{C}$ & & & 07 & $1040-1430$ & C \\
\hline & & 22 & $0750-0930$ & $\mathrm{C}$ & & & 10 & $0900-1050$ & $\mathrm{C}$ \\
\hline & & 23 & 1745 & $\mathbf{M}$ & & & 10 & 0710 & $\mathbf{M}$ \\
\hline & & 24 & 1357 & $\mathbf{M}$ & & & 11 & $0750-1045$ & $\mathrm{C}$ \\
\hline 4 & May & 20 & 0819 & M & 9 & Sept. & .28 & $0745-0910$ & $\mathrm{C}$ \\
\hline & & 21 & $1010-1122$ & $\mathrm{C}$ & & & 29 & 0930 & $\mathbf{M}$ \\
\hline & & 23 & 1755 & $\mathbf{M}$ & & & 30 & $1100-1250$ & $\mathrm{C}$ \\
\hline & & & & & & & 30 & $1400-1455$ & $\mathrm{C}$ \\
\hline 5 & June & 02 & 1505 & $\mathbf{M}$ & & & & & \\
\hline & & 04 & $1300-1420$ & $\mathrm{C}$ & & Oct. & 01 & $0700-0855$ & C \\
\hline & & 04 & 1330 & $\mathbf{M}$ & & & 01 & $1200-1350$ & $\mathrm{C}$ \\
\hline & & 05 & $1200-1245$ & $\mathrm{C}$ & & & 02 & $0735-1000$ & $\mathrm{C}$ \\
\hline & & 05 & 0807 & $\mathbf{M}$ & & & 02 & $1230-1410$ & $\mathrm{C}$ \\
\hline & & 06 & $1255-1340$ & $\mathrm{C}$ & & & 03 & 0730 & $\mathbf{M}$ \\
\hline & & 08 & 1453 & $\mathbf{M}$ & & & 04 & $0625-0900$ & $\mathrm{C}$ \\
\hline & & & & & & & 04 & 0935 & M \\
\hline 6 & June & 19 & 1015 & $\mathbf{M}$ & & & 05 & $0900-1018$ & $\mathrm{C}$ \\
\hline & & 20 & 0920 & $\mathbf{M}$ & & & 05 & 0844 & $\mathbf{M}$ \\
\hline & & & & & & & 05 & 1420 & $\mathbf{M}$ \\
\hline $6 \mathrm{bis}$ & July & 02 & $0630-0657$ & $\mathrm{C}$ & & & 07 & 0750 & $\mathbf{M}$ \\
\hline & & 04 & $0855-1010$ & $\mathrm{C}$ & & & & & \\
\hline & & 05 & $0800-0900$ & $\mathrm{C}$ & 10 & Oct. & 04 & $0920-1120$ & C \\
\hline & & & & & & & 04 & $1200-1327$ & $\mathrm{C}$ \\
\hline 7 & July & 09 & $0500-1530$ & $\mathrm{C}$ & & & 04 & 0927 & $\mathbf{M}$ \\
\hline & & 10 & $0810-0910$ & $\mathrm{C}$ & & & 05 & $1018-1050$ & $\mathrm{C}$ \\
\hline & & 11 & 1200 & $\mathbf{M}$ & & & 05 & 0824 & $\mathbf{M}$ \\
\hline
\end{tabular}

\section{Method of Comparison and General Results}

With a suitable camera we projected the Ca heliograms on the Crimea and Meudon magnetic maps and performed a comparison between plages and magnetic-field structures.

Unfortunately this comparison could be made only for the first transit on the disk of the CSSAR AR.

A comparison between the area within the isogauss lines and the plage areas was 
made using the Crimea magnetic observations only. For all the eleven CSSAR AR the results of these comparisons are examined in Section 3.

Here we notice that the bipolar magnetic regions follow the Hale polarity law. For ARs 2 and 5 belonging to the old 19th cycle the preceding polarity in the Northern hemisphere is North, while for the ARs 1, 3, 4, 6, 6bis, 7, 8, 9, 10, belonging to the even 20th cycle, the preceding polarity in the Northern hemisphere is South.

Often the region of inversion of the magnetic field corresponds to a gap in the plage structure.

Bright patches of plages may coincide with magnetic inclusions, magnetic hills, and occasionally also with regions of inversion of the magnetic field.

The outlines of plage follow well the isogauss of $20-40$ oersted.

\section{Detailed Results of the Comparison}

\section{AR no. 1:}

This is a Northern region of the 20th cycle, visible for two or three transits on the solar disk. It was born on the disk.

It is an extended bipolar region with small peripheral inclusions. The polarity of the preceding part is South.

On March 17 the region of inversion of the magnetic field corresponds to a gap in the plage structure.

On March 18 bright patches of the plage coincide with a region of inversion of the magnetic field and with a magnetic hill.

The isogauss line of 30 oersted follows the outlines of the plage well.

\section{AR no. 3:}

This is a Northern region of the 20th cycle, visible for three transits on the solar disk.

It is a bipolar region with peripheral inclusions. The polarity of the preceding part is South.

The region of inversion of the magnetic field corresponds to a gap on the plage structure.

On May 20 a bright patch of the plage coincides with an inclusion; bright patches of the plage on May 22 and 24 coincide with regions of inversion of the magnetic field.

The isogauss line of 30 oersted follows the outline of the plage well.

\section{AR no. 4:}

This is a Northern region of the 20th cycle, visible for three transits on the solar disk.

It is a bipolar region with very small inclusions. The polarity of the preceding part is South. 
The region of inversion of the magnetic field corresponds to a gap on the plage structure.

On May 21 bright patches of the plage coincide with magnetic hill and an inclusion.

The isogauss line of 30 oersted follows the outline of the plage well.

AR no. 5:

This is a Southern region of the 19th cycle, visible for two transits on the solar disk. It was born on the disk.

It is essentially a bipolar region with peripheral inclusions. The polarity of the preceding part is predominantly South, but in front of the South region there is a small -North region.

The region of inversion of the magnetic field corresponds to a gap in the plage structure.

On June 5 bright patches of the plage coincide with a magnetic hill and an inclusion.

The isogauss line of 40 oersted follows the outline of the plage well.

\section{AR no. 6:}

This is a Southern region of the 20th cycle, visible for three transits on the solar disk. It was born on the disk.

It is a small bipolar region with the two magnetic polarities well separated. The polarity of the preceding part is North. An inclusion of North polarities in the South part is visible.

A bright point of the plage coincides with a magnetic hill; another with an inclusion.

AR no. 6bis:

This is a Northern region of the 20th cycle, visible for one transit. It was born on the disk.

It is a small bipolar region with the two magnetic polarities well separated. The polarity of the preceding part is South.

The region of inversion of the magnetic field corresponds to a gap in the plage structure.

Bright patches of the plage coincide with magnetic hills.

The isogauss line of 40 oersted follows the outline of the plage well.

AR no. 7:

This is a Northern region of the 20th cycle, visible for two transits on the solar disk.

It is a bipolar region. Excluding some very small inclusions, the two magnetic polarities are well separated. The polarity of the preceding part is South.

The region of inversion of the magnetic field corresponds to a gap in the plage structure.

The isogauss line of 20 oersted follows the outline of the plage well. 
AR no. 8:

This is a Northern region of the 20th cycle, visible for two transits on the solar disk.

It is a bipolar region with the two magnetic polarities well separated. The polarity of the preceding part is South.

The region of inversion of one magnetic field corresponds to a gap in the plage structure.

The isogauss lines of $30-40$ oersted follow the outline of the plage well. The increase of area of September $8-11$ is not followed by a corresponding enlargement of the isogauss lines.

AR no. 9:

This is a Northern region of the 20th cycle, visible for three transits on the solar disk. It was born on the disk.

It is substantially a bipolar region, which shows a remarkable inclusion of South polarity during the period of September 28-October 2; on October 5 a small inclusion of South polarity and a North one are present. The polarity of the preceding part is South.

Some parts of the region of inversion of the magnetic field correspond to gaps in the plage structure.

On September 29 and October 2 and 5 bright patches of the plage coincide with inclusions. It is remarkable that on October 3 and 4 bright patches are present in the place where on October 5 the inclusion will appear.

On September 30 and October 4 bright patches of the plage coincide with regions of inversion of the magnetic field.

The isogauss line of 20 oersted follows the outline of the plage well. The sharp increase of the areas within the isogauss between September 28 and 30 is not followed by a corresponding increase of the area of the plage.

\section{AR no. 10:}

This is a Southern region of the 20th cycle, visible for three transits on the solar disk. It was born on the disk on the NW side of an existing region.

It is a complex magnetic region. The polarity of the preceding part is North with remarkable inclusions of South polarities.

For October 4 the plage shows gaps coinciding with regions of inversions of the magnetic field. On October 5 these gaps are no longer visible, perhaps because of the nearness of the plage to the Sun's West limb.

Brightenings of the plage coincides with inclusions.

The isogauss line of 20 oersted follows the outline of the plage well.

\section{The Histograms of Distances of Magnetic-Field Intensity Peaks}

Looking at the magnetic maps of the CSSAR active regions we may see in the 
distribution of magnetic fields the very well pronounced characteristic structure of the supergranular network (see e.g. Figures 1 and 2). Therefore one may expect that from the measurements of distances of individual peaks of magnetic-field intensity in each active region, one will obtain the same characteristic values as were found from measurements of Ca plages (Rogerson, 1955; Simon and Leighton, 1964).

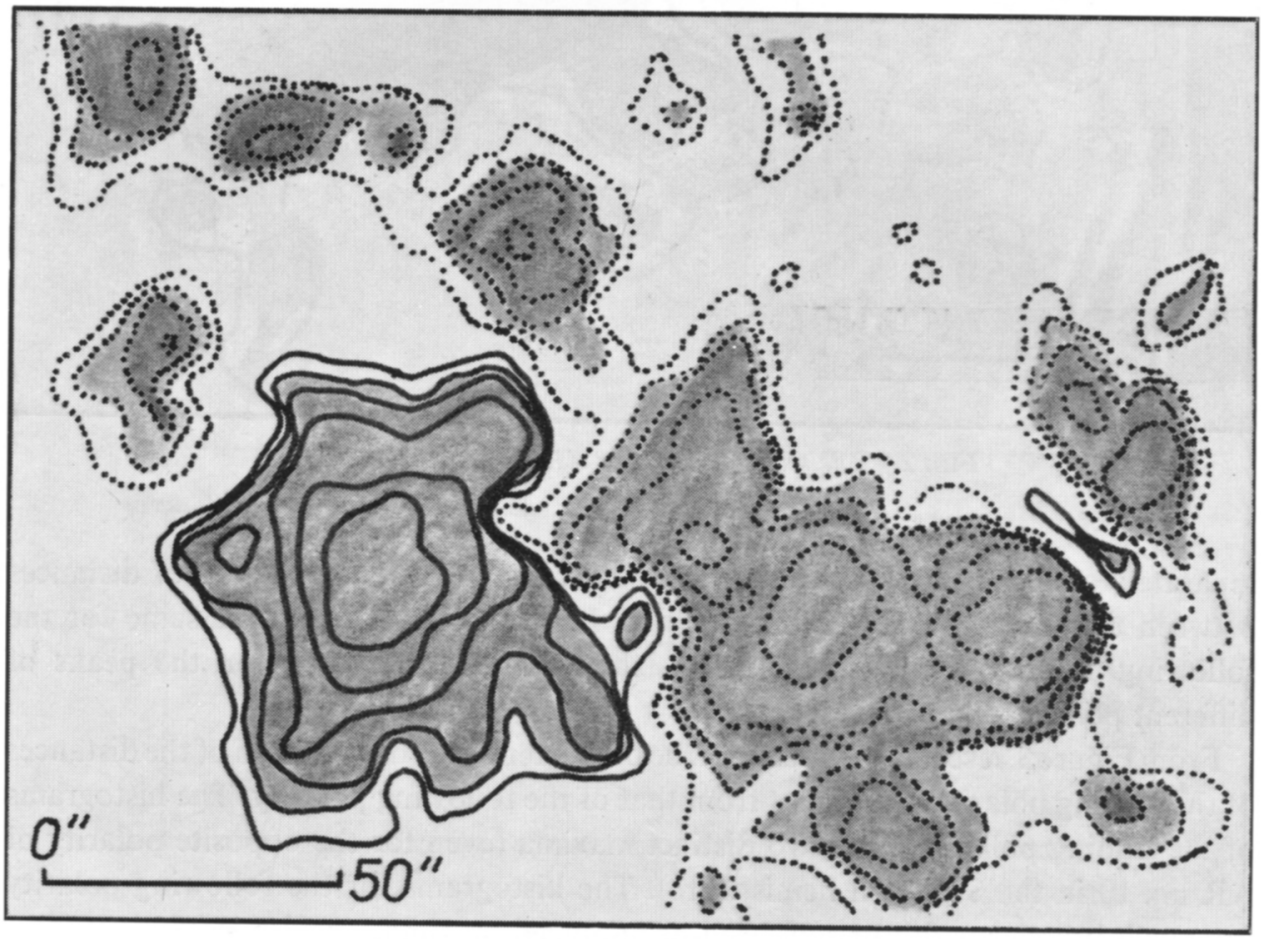

FIG. 1. Crimean magnetic map of the longitudinal component of the photospheric magnetic field for AR no. 7 (July 7, 1965). The positive polarity is indicated by solid lines, the negative polarity by dotted lines. Areas with intensity $\mathrm{H} \geqslant 20$ gauss are shaded.

For these measurements of distances between magnetic-field intensity peaks, the 38 Crimean magnetic maps were used. The distances were estimated between all peaks with intensity greater than 20 gauss - separately between plus-plus peaks, minus-minus peaks and plus-minus peaks. We took a value of $100^{\prime \prime}$ as an upper limit for the distance. The total number of measurements exceeded 3000 individual values.

The main results in the form of histograms of measured values are presented in Figure 3. Because of the small number of individual peaks in some active regions, measured values obtained for smaller active regions are combined in one histogram (Figures 3a, d, g). The histograms of the largest active region, No. 9, are presented 


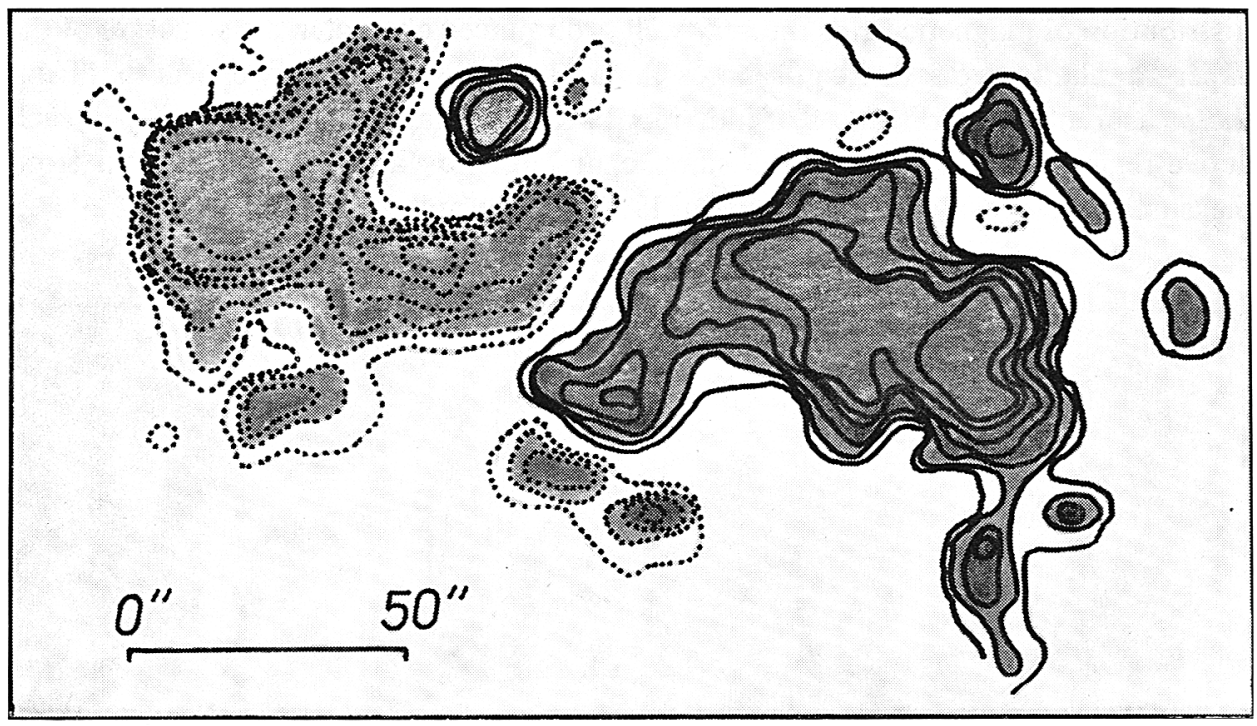

FIG. 2. The magnetic map for AR no. 5 (June 4, 1965).

separately as for region No. 10 . Figures $3 a, b, c$ show the distribution of distances between the peaks of the leading polarity, Figures 3d, e, f show the same for the following polarity, and Figures $3 \mathrm{~g}, \mathrm{~h}$, i, show the distances between the peaks of different polarities.

From Figure 3, it seems obvious that the character of the distribution of the distances of the leading polarity is different from that of the following polarity. The histograms of the leading polarity show two distinct maxima (even for the opposite polarity of AR no. 10 in the Southern hemisphere). The histograms for the following polarity are much broader with not such well-pronounced maxima.

The values of the characteristic distances of the leading polarity magnetic-field intensity peaks $\left(30^{\prime \prime}-40^{\prime \prime}\right.$ and $\left.60^{\prime \prime}-70^{\prime \prime}\right)$ coincide well with the values found for the supergranular network (Rogerson, 1955; Simon and Leighton, 1964), as well as with the values estimated for the distances of the feet of quiescent filaments (Sýkora, 1967). These facts may have some importance for the future study of differences between the roles of the leading and following polarities in solar activity development.

The smallest value of the distances between the magnetic peaks of the leading and following polarities which corresponds to about $30^{\prime \prime}-35^{\prime \prime}$, also seems to be of interest.

\section{Acknowledgements}

We are indebted to Dr. Michard, to Mrs. Martres and to the other colleagues of the Meudon Solar Service for their kindness and help during our stay at Meudon. 

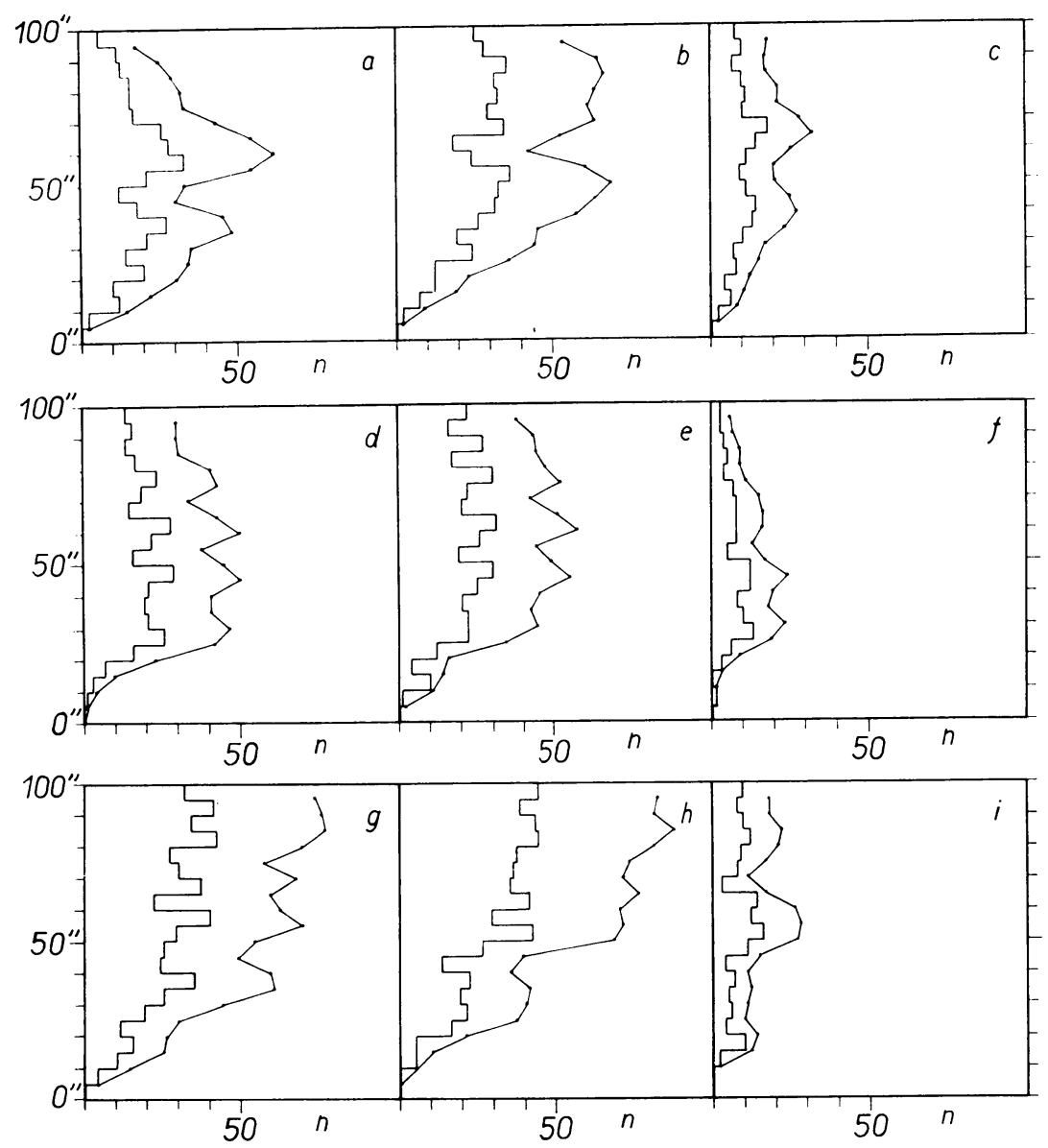

FIG. 3. Histograms of the distances between the peaks of the photospheric magnetic-field longitudinal component ( $H \geqslant 20$ gauss) for CSSAR Crimean magnetic maps. On the vertical axis the distances are in seconds of arc; on the horizontal axis the frequency of individual values is given. The curves for the summation of values of distance in two intervals: $5^{\prime \prime}$ and 10" are drawn. The curves $a, b, c$ represent the measurements of distances of peaks of the leading polarity (a) for ARs no. 1, 3, 4, 6bis, 7, 8, (b) for $A R$ no. 9 all from the Northern hemisphere, (c) for AR no. 10 from the Southern hemisphere. The curves $d, e, f$ show the same measurements for the following polarities respectively. The curves $g, h, i$ are, in the same order, histograms of distances of peaks of different polarities.

\section{References}

Godoli, G., Monsignori Fossi, B.C. (1968) in the present volume, p. 326.

Howard, R. (1959) Astrophys. J., 130, 193.

Rogerson, Jr., J. (1955) Astrophys. J., 121, 204.

Simon, G.W., Leighton, R. B. (1964) Astrophys. J., 140, 1120.

Sýkora, J. (1967) Bull. astr. Obs. Csl. (in print).

Tsap, T.T. (1967) Izv. krym. astrofiz. Obs. (in print). 